\title{
Teratoma showing the features of retinal structure: A case of sacrococcygeal teratoma
}

\author{
MANABU TAKAMATSU ${ }^{1}$, HITOMI AOKI ${ }^{2}$, YOSHINOBU HIROSE ${ }^{1}$, KAZUHIRO KOBAYASHI $^{1}$, \\ HIROYUKI TOMITA $^{1}$, TOSHIYA KUNO ${ }^{1}$, HISASHI KOUMURA ${ }^{3}$ and AKIRA HARA ${ }^{1}$ \\ Departments of ${ }^{1}$ Tumor Pathology, and ${ }^{2}$ Tissue and Organ Development, Gifu University Graduate School of Medicine, \\ Gifu 501-1194; ${ }^{3}$ Department of Pediatric Surgery, Nagara Medical Center, \\ National Hospital Organization, Gifu 502-0071, Japan
}

Received December 30, 2011; Accepted February 29, 2012

DOI: $10.3892 / \mathrm{ol} .2012 .636$

\begin{abstract}
Teratoma is a tumor that forms triploblastic tissues and the common sites of occurrence are sacrococcygeal lesions and the ovaries. The majority of cases are curable with surgical resection and the prognosis depends on the extent and histological scoring of the tumor. In the present study, we report a case of sacrococcygeal teratoma of a newborn showing features of a retina-like structure. A 29-year-old woman gave birth prematurely to an infant girl with sacrococcygeal teratoma. Surgical resection was performed 10 days after delivery. The tumor contained immature components as well as a retina-like structure. Several investigations, including immunohistochemical analysis, confirmed the similarities between the normal mouse retina and the retina-like structure of the tumor. The vascular arrangement and polarity surrounding the retina-like structure are unique and this is thought to be significant in the induction of structural differentiation. Our findings may provide insights into the matter of teratogenic activity in stem cell therapies for clinical applications.
\end{abstract}

\section{Introduction}

Teratoma is a tumor of embryonic origin, composed of various tissues derived from three germ layers (ectoderm, endoderm and mesoderm), which differentiate to form somatic tissues (1). The teratoma may comprise either well-differentiated (mature) or primitive (immature) tissue. The tumor is diagnosed as an immature teratoma if there are areas of tissue immaturity in the stromal, epithelial or neural component. The ectodermal components typically include squamous epithelium and neural tissue. Although neural tissue is usually a major component

Correspondence to: Dr Manabu Takamatsu, Department of Tumor Pathology, Gifu University Graduate School of Medicine, 1-1 Yanagido, Gifu 501-1194, Japan

E-mail: sw20.3s-gte@hotmail.co.jp

Key words: sacrococcygeal, teratoma, retina of immature and mature teratoma, a retinal structure is rarely identified in teratoma tissues.

Sacrococcygeal teratoma appears on the caudal portion of the newborn and accounts for $40 \%$ of all teratomas in the first two decades of life, followed by ovarian teratoma (37\%) (2). The incidence of the disease is between $1 / 35,000$ and $1 / 40,000$ live births and has a 4:1 female:male ratio (3). Almost all cases of sacrococcygeal teratoma are diagnosed in the perinatal period, in contrast to ovarian teratoma which are rare within the first 24 months of life and seldom appear prior to the age of 6 years (2). Prenatal examination, including abdominal ultrasonography, may detect the sacrococcygeal mass, which is treated with surgical resection following birth (4). The prognosis depends on the extent and histological scoring of the tumor, with an excellent prognosis if the tumor has no immature components and adequate resection is performed (3).

In the present study, we report a case of sacrococcygeal teratoma including the features of a retina-like structure. The similarities between the retina-like structure within the teratoma and the normal mammalian retina are investigated with regard to tissue structure and protein expression (5).

The study was conducted with the approval of the Ethics Committee of the University of Gifu, and consent was obtained from the patient and her family.

\section{Patients and methods}

Clinical history. Ultrasonic examination revealed a mass on the buttocks of a fetus within the uterus of a 29-year-old woman. At 28 weeks of pregnancy, magnetic resonance imaging (MRI) revealed that the mass had solid, cystic components and it appeared that the majority of the tumor was located outside of the fetal body (Fig. 1A). One week later, a Caesarean section was performed as the fetus was showing signs of distress. The newborn was premature (29 w, 3 d), of low birth weight $(1,152 \mathrm{~g})$ and had a mass on her back. Following several treatments during the perinatal period, including intratracheal intubation and surfactant therapy for respiratory distress syndrome (RDS), surgical resection was performed 10 days after delivery. The majority of the mass appeared to be located outside of the pelvis and was completely resected. Analysis of the peripheral blood of the newborn revealed an elevation 
A

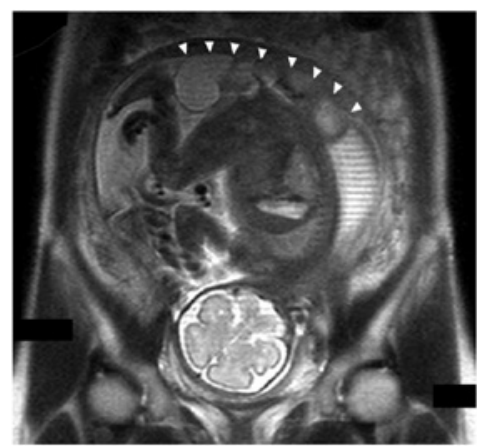

B

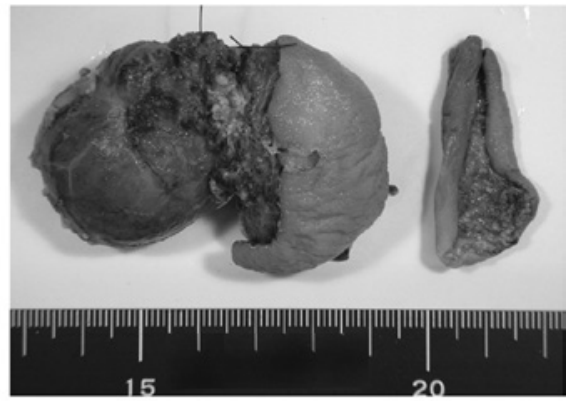

C

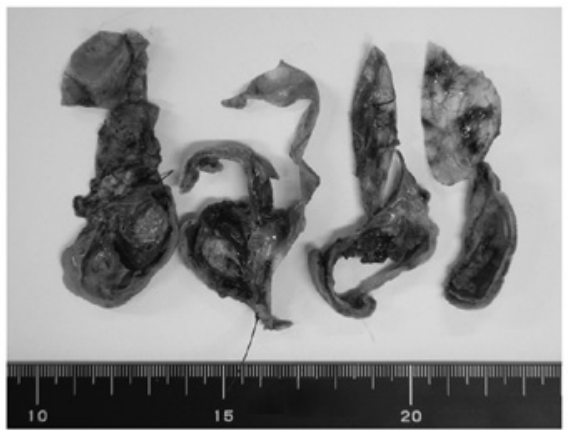

Figure 1. Macroscopic features of the sacrococcygeal teratoma. (A) Coronal section of the fetus in the uterus using magnetic resonance imaging (MRI). The mass on the bottom of the fetus is indicated by white arrowheads. (B) Whole image of the tumor and (C) cross-sectional surface. The tumor had dark-yellow solid components and cystic lesions.

in the $\alpha$-fetoprotein level at birth $(680,000 \mathrm{ng} / \mathrm{ml})$, which then decreased eight weeks after surgery $(63,000 \mathrm{ng} / \mathrm{ml})$. The newborn experienced no major problems following surgery and was discharged from the hospital when she had a body weight of $2,369 \mathrm{~g}$.

Tissue sections and staining. The tissue specimens were formalin-fixed and paraffin-embedded. The tissue sections were stained with hematoxylin and eosin (H\&E), Kluver-Barrera staining and silver staining. To determine the cell types of the retina-like structure of the teratoma, we also used immunohistochemical staining with monoclonal or polyclonal antibodies against pax6 (1:100, Chemicon, Temecula, CA, USA), synaptophysin (1:200 Dako, Carpinteria, CA, USA), $\beta$-tubulin (1:1000 Covance, Princeton, NJ, USA), Hu C/D (1:100 Molecular Probes, Carlsbad, CA, USA), Nestin (1:400 Chemicon), GFAP (1:300 Dako), RPE 65 (1:200 Millipore, Billerica, MA, USA), HMB45 (1:200 Dako) and Ki-67 (1:200 Dako).

\section{Results}

Macroscopic observations. Macroscopically, the mass had a grayish tense cyst $3 \mathrm{~cm}$ in diameter and slightly hard components covered with skin (Fig. 1B). On the cut surface, the cyst had a thin wall with scattered petechial hemorrhagic lesions and the other section contained solid dark-yellow components with thick walls that also contained a cystic lesion (Fig. 1C).

Histological observations. Histologically, the cysts were covered with fibrous tissue and the solid components contained glandular, brain, neural, epithelial, cartilaginous and adipose tissues (Fig. 2A, B and E-L). The mass also had primitive neuroepithelium consisting of small hyperchromatic cells arranged in rosettes (Fig. 2C). Consequently, the infant was diagnosed as having mature cystic teratoma containing immature components. In one section of the capsules, we observed cells arranged linearly along a fibrous membrane which had a multilayer structure resembling the retina (Fig. 2D).

Comparison with normal mouse retina. There are several cell types in the normal mouse and human retina, including ganglion, bipolar and photoreceptor cells. The retina-like structure in the teratoma of the present case (Fig. 3 capital letters) and a mouse retina as a control (Fig. 3 small letters) are shown with several methods of staining. In H\&E staining, the retina-like structure of the teratoma (Fig. 3A) shows three cell layers, which resemble the ganglion cell and inner and outer nuclear layers in the normal mouse retina (Fig. 3a). The fine fibrous layer, which resembles optic nerve fibers and was positive for nestin (Fig. 3G) and GFAP (Fig. 3H), is connected to the layer with irregularly arranged nuclei, which resembles the ganglion cell layer and was positive for Pax6 (Fig. 3B) and $\mathrm{HuC} / \mathrm{D}$ (Fig. 3F). The second layer has dense cells with small, round nuclei and scanty cytoplasm which were focally positive for Pax6 (Fig. 3B), synaptophysin (Fig. 3C), $\beta$-tubulin (Fig. 3D) and HuC/D (Fig. 3F), which is similar to the inner nuclear layer of the mouse retina. The cells of the nuclear layer opposite to the stromal tissue have nuclei that are similar to those of photoreceptors and were positive for synaptophysin (Fig. 3C). There were no clear cones and rods connected to the photoreceptor cell bodies (Fig. 3A, E and I). Among the three cell layers, we observed a bundle of fine eosinophilic fibers resembling the inner and outer plexiform layers.

The retinal pigment epithelium, which is positive for RPE65 and HMB45 in the normal mouse retina (Fig. 3j and k), was not identified in the retina-like structure of the teratoma 

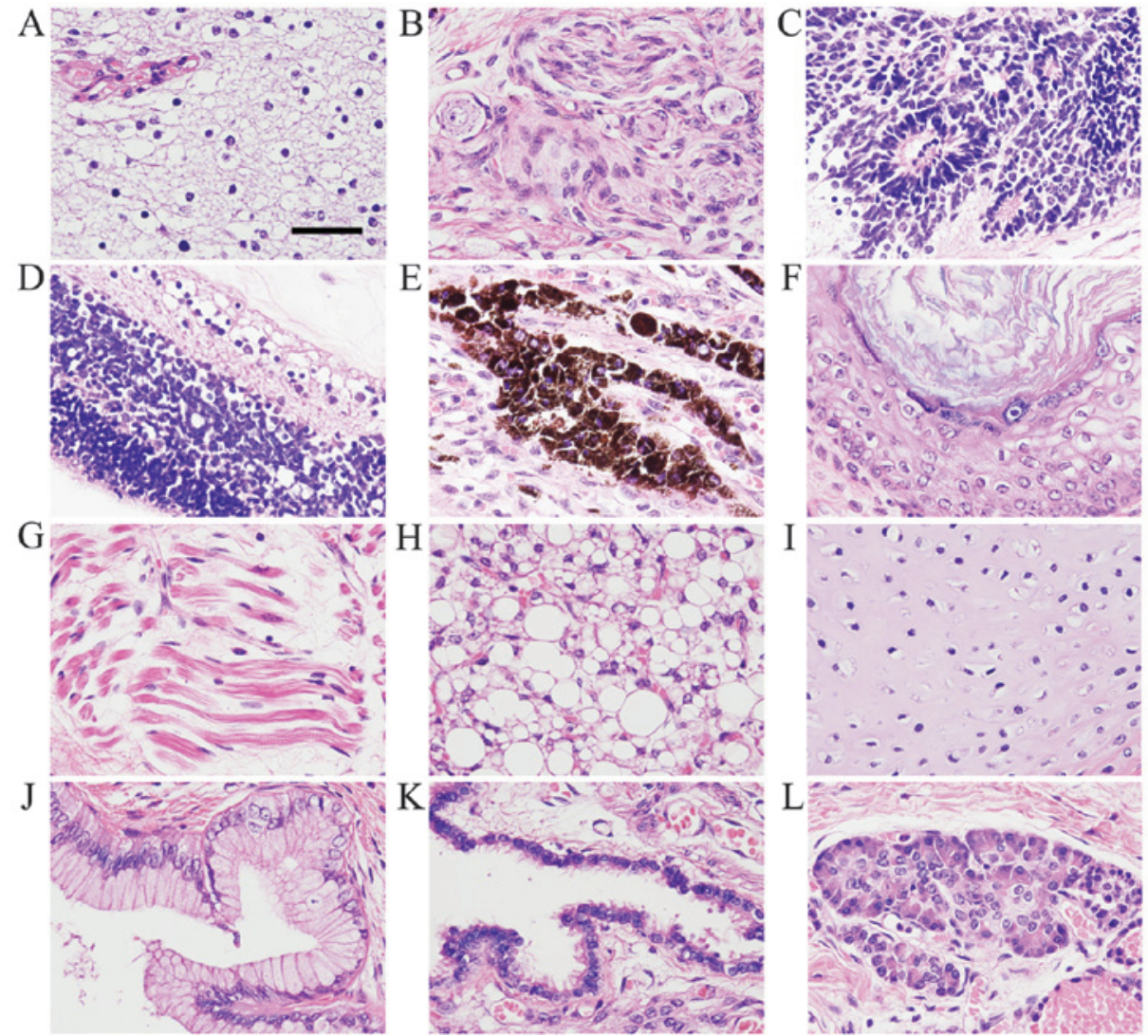

Figure 2. Histopathological findings of the sacrococcygeal teratoma. The teratoma contained tissues from three germ layers: ectoderm, mesoderm and endoderm. The ectodermal tissues of the teratoma include brain tissue comprising (A) glia cells, (B) peripheral nerve with ganglion cells, (C) primitive neuroepithelium consisting of small hyperchromatic cells arranged in rosettes, (D) retina-like structure, (E) pigment epithelium containing melanin granules and (F) squamous epithelium with keratinization. The mesodermal tissues of the teratoma include $(\mathrm{G})$ striated muscle fibers, $(\mathrm{H})$ adipose tissue and (I) hyaline cartilage. The endodermal tissues include (J) columnar epithelium, (K) respiratory ciliated epithelium and (L) secretory gland-like structure. Hematoxylin and eosin (H\&E) staining. Scale bar in (A), $30 \mu \mathrm{m}$.
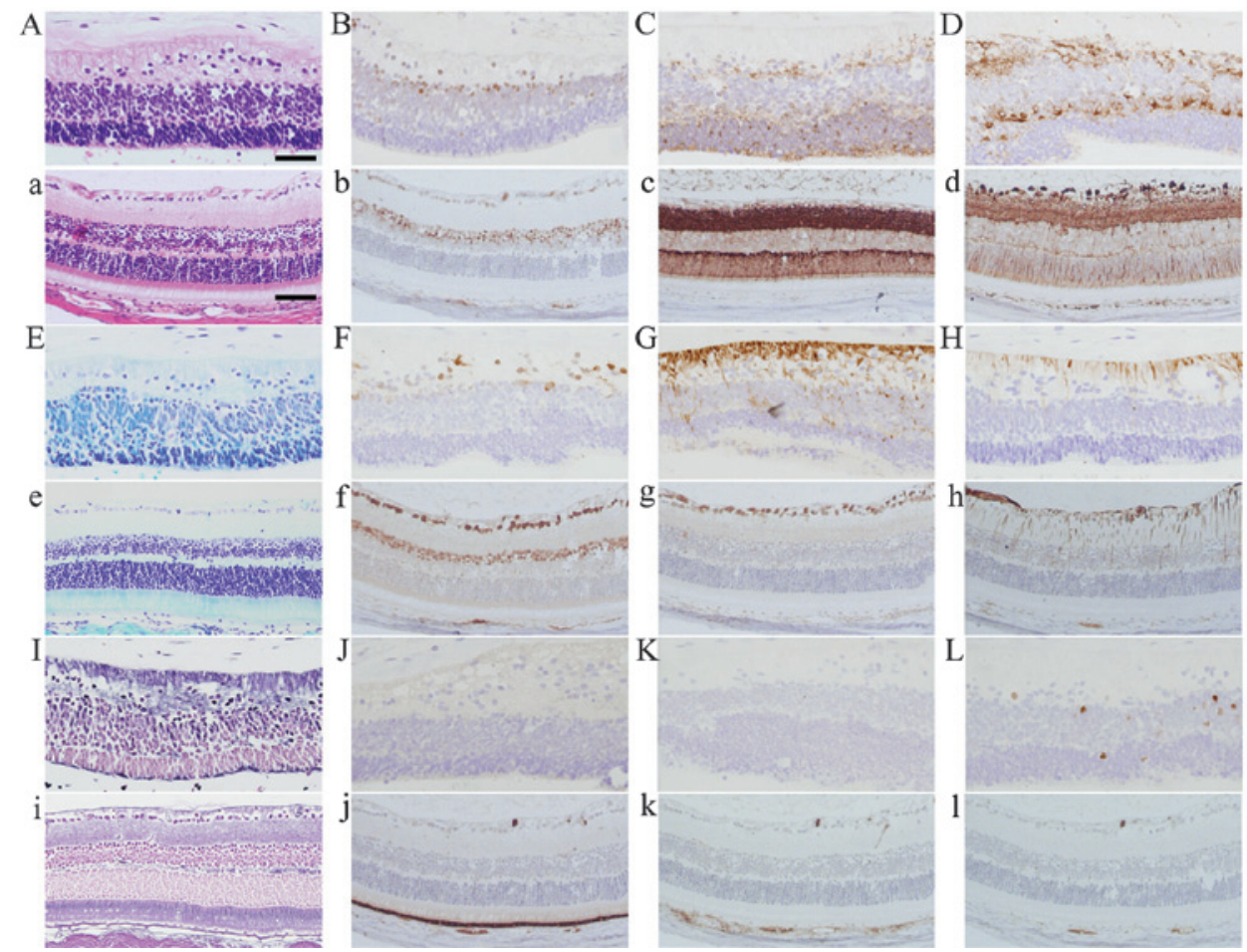

Figure 3. Histopathological images of the retina-like structure in the human teratoma (A-L) with a normal mouse retina as a control (a-1). (A, a) H\&E staining, (E, e) Kluver-Barrera staining, (I, i) silver staining. Immunohistochemistry for (B, b) Pax6, (C, c) synaptophysin, (D, d) $\beta$-tubulin, (F, f) HuC/D, (G, g) nestin, (H, h) GFAP, (J, j) RPE65, (K, k) HMB45 and (L, 1) Ki-67. Scale bars in (A) $50 \mu \mathrm{m}$; in (a), $75 \mu \mathrm{m}$. 
(Fig. 3J and K). There were few proliferating cells positive for $\mathrm{Ki}-67$ in the retina-like structure of the teratoma and these cells were not observed in the normal mouse retina.

\section{Discussion}

Although tumors located in the sacrococcygeal region have been reported since the 19th century, the first review to elaborate on sacrococcygeal teratoma was by Deveer and Browder in 1937 (6). The American Academy of Pediatrics Surgical Section (AAPSS) classified sacrococcygeal teratomas into four groups according to the extent of the tumor and its histological appearance (3). The extent of the tumor is described as follows: Type I, predominantly external with minimal presacral component; Type II, external but with significant intrapelvic extension; Type III, apparent externally but predominantly a pelvic mass extending into the abdomen; Type IV, presacral with no external components (7). The histological findings are graded as follows: Grade 0, tumor contains only mature tissue; Grade 1, tumor contains rare foci of immature tissues; Grade 2, tumor contains moderate quantities of immature tissues; Grade 3, tumor contains large quantities of immature tissue with or without malignant yolk sac elements (8). The present case was diagnosed as Type I and Grade 1 according to the AAPSS classification.

Teratomas with a retina-like structure have been reported and some of the cases showed a complete or partial eye structure (9). A retrospective search in our medical center revealed that 2 out of 7 cases of sacrococcygeal teratoma of newborns had retina-like structures; one is the present case and the other showed an incompletely stratified retinal structure associated with retinal markers. The two cases also had immature neural components, including true rosettes and primitive neural tubes. However, 4 out of 40 cases of ovarian teratoma had immature neural components and no retinal structure was found in the tissues. These observations indicate that the immature components of teratomas are associated with the formation of the retina-like structure and that the occurrence of the teratoma at the sacrococcygeal site is significant in the induction of a retinal structure.

Results of our histological and immunohistochemical analysis reveal that a retina-like structure of the teratoma resembled almost all of the characteristics of normal retina, with the exception of rods and cones. To form such a structure, there must be certain factors which polarize and differentiate the cells. In the teratoma, stromal tissue, including arteries feeding the retina-like structure, was connected to the side of the ganglion cell layer, which is the same as the normal retina fed by the central retinal artery. Moreover, the stromal tissue adjacent to the retina-like structure had only a few vessels, in contrast to that of the less differentiated or immature neural components. This observation shows that polarized structures, including the normal retina and retina-like structure, need a gradient of concentration of oxygen or blood flow during formation and structural development, especially under hypoxic conditions (10). In addition, retinopathy of prematurity is thought to be the result of excessive oxygen exposure (11) and may be relevant to the formation of proliferative lesions, since varying oxygen concentrations may influence microvascular formation. A precise and careful search of more cases is needed to clarify the hypothesis of retinal differentiation within the teratoma.

In conclusion, we have reported a case of sacrococcygeal teratoma with a retina-like structure in a newborn and suggested the importance of blood supply and oxygen circumstance in the formation of the stratified structure. This finding may aid the development of efficient methods to solve the problem of condition setting in retinal transplantation (12-14) using embryonic stem (ES) cell (15) or induced pluripotent stem (iPS) cell (16) technology, which may cause teratogenic transformation.

\section{References}

1. Azizkhan RG and Caty MG: Teratomas in childhood. Curr Opin Pediatr 8: 287-292, 1996.

2. Tapper D and Lack EE: Teratomas in infancy and childhood. A 54-year experience at the Children's Hospital Medical Center. Ann Surg 198: 398-410, 1983.

3. Tuladhar R, Patole SK and Whitehall JS: Sacrococcygeal teratoma in the perinatal period. Postgrad Med J 76: 754-759, 2000.

4. Winderl LM and Silverman RK: Prenatal identification of a completely cystic internal sacrococcygeal teratoma (type IV). Ultrasound Obstet Gynecol 9: 425-428, 1997.

5. Aoki H, Hara A, Niwa M, Yamada Y and Kunisada T: In vitro and in vivo differentiation of human embryonic stem cells into retina-like organs and comparison with that from mouse pluripotent epiblast stem cells. Dev Dyn 238: 2266-2279, 2009.

6. Deveer JA and Browder J: Sacrococcygeal teratoma. Ann Surg 105: 408-417, 1937.

7. Murphy JJ, Blair GK and Fraser GC: Coagulopathy associated with large sacrococcygeal teratomas. J Pediatr Surg 27: 1308-1310, 1992.

8. Graf JL, Housely HT, Albanese CT, Adzick NS and Harrison MR: A surprising histological evolution of preterm sacrococcygeal teratoma. J Pediatr Surg 33: 177-179, 1998.

9. Sergi C, Ehemann V, Beedgen B, Linderkamp O and Otto HF: Huge fetal sacrococcygeal teratoma with a completely formed eye and intratumoral DNA ploidy heterogeneity. Pediatr Dev Pathol 2: 50-57, 1999.

10. Claxton S and Fruttiger M: Oxygen modifies artery differentiation and network morphogenesis in the retinal vasculature. Dev Dyn 233: 822-828, 2005.

11. Zhang W, Ito Y, Berlin E, Roberts R and Berkowitz BA: Role of hypoxia during normal retinal vessel development and in experimental retinopathy of prematurity. Invest Ophthalmol Vis Sci 44: 3119-3123, 2003.

12. Hara A, Niwa M, Kunisada T, et al: Embryonic stem cells are capable of generating a neuronal network in the adult mouse retina. Brain Res 999: 216-221, 2004.

13. Hara A, Niwa M, Kumada M, et al: Intraocular injection of folate antagonist methotrexate induces neuronal differentiation of embryonic stem cells transplanted in the adult mouse retina. Brain Res 1085: 33-42, 2006

14. Hara A, Taguchi A, Aoki H, et al: Folate antagonist, methotrexate induces neuronal differentiation of human embryonic stem cells transplanted into nude mouse retina. Neurosci Lett 477: 138-143, 2010.

15. Sadowski D, Kiel ME, Apicella M, Arriola AG, Chen CP and McKinnon RD: Teratogenic potential in cultures optimized for oligodendrocyte development from mouse embryonic stem cells. Stem Cells Dev 19: 1343-1353, 2010.

16. Kooreman NG and Wu JC: Tumorigenicity of pluripotent stem cells: biological insights from molecular imaging. J R Soc Interface 7 (Suppl 6): S753-S763, 2010. 